\title{
Equity Trading Volume and Volatility: Latent Information Arrivals and Common Long-Run Dependencies
}

Tim BollersLeV

Department of Economics, Duke University, Durham NC 27708 (boller@econ.duke.edu)

\section{Dan JUBINSKI}

Department of Economics, University of Virginia, Charlottesville, VA 22901 (pdj7x@virginia.edu)

\begin{abstract}
This article examines the behavior of equity trading volume and volatility for the individual firms composing the Standard \& Poor's 100 composite index. Using multivariate spectral methods, we find that fractionally integrated processes best describe the long-run temporal dependencies in both series. Consistent with a stylized mixture-of-distributions hypothesis model in which the aggregate "news"-arrival process possesses long-memory characteristics, the long-run hyperbolic decay rates appear to be common across each volume-volatility pair.
\end{abstract}

KEY WORDS: Fractional integration; Mixture of distributions hypothesis; Return volatility; Spectral analysis; Trading volume.

An extensive empirical literature has developed over the past decade for modeling the temporal dependencies in financial-market volatility. A common finding to emerge from most of these studies concerns the extremely high degree of own serial dependencies in the time series of absolute or squared returns and/or the estimated volatility processes. Bollerslev, Chou, and Kroner (1992) discussed over 200 of the earliest studies in this literature using autoregressive conditional heteroscedasticity (ARCH)-type models, and Ghysels, Harvey, and Renault (1996) provided a more recent survey of the literature on stochastic volatility models. Very little theoretical work has explored the structural determinants behind these dynamic dependencies.

Meanwhile, numerous empirical studies have documented the existence of a strong positive contemporaneous correlation between trading volume and volatility [see Karpoff (1987) for an early discussion of this literature]. This positive correlation is consistent with most theoretical market microstructure models involving the strategic interaction among asymmetrically information-rational agents [see O'Hara (1995) for a survey of the relevant literature]. Unfortunately, these same theoretical models generally remain very vague, if not silent, about the long-run dynamic relationship between trading volume and volatility. A less structural approach for rationalizing the strong contemporaneous correlation between trading volume and volatility is provided by the so-called mixture-of-distributions hypothesis (MDH) pioneered by Clark (1973), Epps and Epps (1976), and Tauchen and Pitts (1983). According to the $\mathrm{MDH}$, returns and trading volume are driven by the same underlying latent "news"-arrival, or information-flow, variable so that the arrival of unexpected "good news" results in a price increase, whereas "bad news" results in a price decrease. Both of these events are accompanied by above-average trading activity in the market as it adjusts to a new equilibrium. Accordingly, the absolute returns, or volatility, and trading volume should be positively correlated.

Although the first generation of tests of the MDH by Harris $(1986,1987)$ and others were generally supportive of the model, subsequent studies by Lamoureux and Lastrapes (1994) and Richardson and Smith (1994) indicated that the same latent information-arrival process is unable to describe the short-run dynamic dependencies in both equity trading volume and volatility. More recently, however, Andersen (1996) argued that these apparent rejections of the MDH may be due to artificial and unwarranted distributional assumptions. Building on the Glosten and Milgrom (1985) market microstructure setting in which a riskneutral market maker posts competitive bid and ask prices, the resulting modified MDH with Poisson-distributed trading volume is not rejected for the same individual common shares that firmly reject the more traditional version of the $\mathrm{MDH}$, assuming trading volume to be normally distributed. Meanwhile, the estimated joint latent information arrival processes indicate a surprisingly low degree of volatility persistence compared to the empirical results reported in the extant ARCH and stochastic volatility literature. In fact, this latter body of literature has led several studies (see, among others, Dacorogna, Müller, Nagler, Olsen, and Pictet 1993; Ding, Granger, and Engle 1993; Baillie, Bollerslev, and Mikkelsen 1996; Bollerslev and Mikkelsen 1996; Granger and Ding 1996; Robinson and Zaffaroni 1997; Andersen and Bollerslev 1997a; Breidt, Crato, and de Lima 1997; Lobato and Savin 1998; Ray and Tsay 1998) to the conclusion that

(C) 1999 American Statistical Association Journal of Business \& Economic Statistics January 1999, Vol. 17, No. 1 
the second-order moment dynamics of many return series are best characterized by fractionally-integrated-type processes.

The available empirical evidence regarding the own dynamic dependencies in financial-market trading volume is more limited in its scope. Still, the sample autocorrelations for the portfolio turnover ratios reported by Lo and Wang (1996) and the seminonparametric density estimates for aggregate stock-market index returns and trading volume reported by Gallant, Rossi, and Tauchen $(1992,1993)$ and the corresponding findings for individual stocks by Tauchen, Zhang, and Liu (1996) all point toward the existence of long-term dynamic dependencies in equity trading volume. As such, the aforementioned conflicting empirical results based on the standard MDH and the assumption of a single latent information-arrival process may simply reflect the existence of a more complex dynamic relationship between trading volume and volatility. Although the longrun dependencies are common, the short-run responses to certain types of "news" are not necessarily the same across the two variables. This idea also motivates the bivariate MDH specification, recently estimated by Liesenfeld (1998a,b).

This article adds to this extant literature by testing implications of the MDH as a long-run proposition in which the aggregate latent information-arrival process possesses long-memory characteristics. Consistent with this formulation, the 100 log-periodogram estimates for the degree of fractional integration, or $d$, for the absolute returns for each of the 100 individual common shares in the Standard and Poor's 100 (S\&P100) composite index generally put the degree of fractional integration in the interval between 0 and $\frac{1}{2}$. Thus, although the individual equity volatility processes are highly persistent, they appear to be covariance stationary so that all "news" shocks eventually dissipate, albeit at a very slow hyperbolic rate of decay. Applying this same seminonparametric estimation procedure to the 100 individual equity trading-volume series results in equally significant long-memory characteristics. No prior estimates to this effect are available in the existing literature. The close pairwise correspondence between the estimates for $d$ across each of the 100 volume-volatility series is particularly noteworthy, and formal hypothesis tests do not, in general, reject that the two series possess a common longrun hyperbolic decay rate. As such, these empirical findings support the notion of the MDH as a long-run phenomenon driven by a slowly mean-reverting fractionally integrated latent information-arrival process.

The remainder of the article is organized as follows. Section 1 sets out the notation and briefly reviews the arguments behind the standard MDH, along with the particular structure leading to common long-memory volatility and volume dependencies. The econometric techniques employed in estimating and testing for a common degree of fractional integration, as formally developed by Robinson (1995), are discussed in Section 2. The complete estimation results for all of the 100 individual stocks included in the S\&P100 composite index are discussed in Section 3. For illustrative purposes, this section also details the em- pirical results as they relate to Aluminum Corporation of America (AA), the alphabetically first, by ticker symbol, of the 100 common shares. Section 4 provides a few concluding remarks. An appendix provides detailed sensitivity analysis.

\section{LATENT INFORMATION ARRIVALS AND LONG-MEMORY DEPENDENCIES}

To set out our notation, let $R_{j, t}, t=1,2, \ldots, T$ and $j=1,2, \ldots, N$, denote the daily continuously compounded return on stock $j$ corrected for any dividend payments and/or stock splits. The corresponding daily trading volume will be denoted by $V_{j, t}$.

\subsection{Return and Volume Dynamics}

The daily returns are naturally decomposed into a sum of the intradaily logarithmic equilibrium price changes. Assuming that these logarithmic price changes are iid with a finite variance and that the number of such pseudo intradaily equilibria are "large," it follows by a standard central limit theorem argument that, conditional on the number of price changes, the daily returns should be normally distributed. The assumption of iid price changes also implies that the returns should be serially uncorrelated through time. Of course, the actual number of intradaily price changes is likely to vary across time, depending on the number, or intensity, of the "news" arrivals that occur during the day. Conditional on this latent intensity process, say $K_{j, t}$, the distribution for the daily returns may be expressed as

$$
R_{j, t} \mid K_{j, t} \sim \mathrm{N}\left(0, \sigma_{j}^{2} \cdot K_{j, t}\right),
$$

where $K_{j, t}$ has been standardized so that a value of unity will result in a daily variance for stock $j$ equal to $\sigma_{j}^{2}$. This particular representation for the returns and the underlying arguments mirror the original derivation of the MDH by Clark (1973).

In this standard formulation of the $\mathrm{MDH}$, the $K_{j, t}$ latent information-arrival process represents the intensity of both firm-specific and marketwide "news" events. As such the $K_{j, t}$ 's will almost certainly be highly contemporaneously correlated. Most tests and empirical implementations of the MDH have invariably ignored this aspect of the model. We shall not pursue this commonality in the present analysis either, although future work along these lines may result in important efficiency gains and new insights.

Instead we now turn to the basic implications of the MDH for the joint trading volume-volatility relationship. Characterizing the sequence of pseudo intradaily equilibria as arising from the strategic interaction of informed investors and liquidity traders in a Glosten and Milgrom (1985) framework with a risk-neutral market maker, Andersen (1996) showed that the resulting daily trading volume, conditional on the information-arrival process, will be approximately Poisson distributed. Specifically,

$$
V_{j, t} \mid K_{j, t} \sim \operatorname{Po}\left(\mu_{j, 0}+\mu_{j, 1} \cdot K_{j, t}\right),
$$


where $\mu_{j, 0}$ and $\mu_{j, 1}$ are normalizing constants related to the importance of liquidity, or noise, and information-based trading, respectively. The Poisson distribution guarantees that trading volume is never negative, and the liquidity trading constant $\mu_{j, 0}$ allows for a more flexible nonproportional relationship, in comparison to the standard MDH with $\mu_{j, 0} \equiv 0$ and conditionally normally distributed trading volume.

The basic tenets of the MDH remain intact, however. In particular, it follows that, for the modified MDH in Equations (1) and (2),

$$
\operatorname{cov}\left(R_{j, t}, V_{j, t}\right)=0
$$

and

$$
\begin{aligned}
\operatorname{cov}\left(\left|R_{j, t}\right|, V_{j, t}\right) & \\
= & (2 / \pi)^{1 / 2} \cdot \mu_{j, 1} \cdot\left[E\left(K_{j, t}^{3 / 2}\right)-E\left(K_{j, t}^{1 / 2}\right)\right]>0 .
\end{aligned}
$$

Whereas the returns, $R_{j, t}$, and the contemporaneous trading volume, $V_{j, t}$, are uncorrelated, the absolute returns, $\left|R_{j, t}\right|$, and trading volume are positively correlated. Of course, this contemporaneous correlation between trading volume and volatility does not reflect a causal relationship but rather the subordination to the same latent information-arrival process, $K_{j, t}$.

\subsection{Latent Information-Arrival Dynamics}

Although most of the earlier investigations of the MDH, as exemplified by the work of Epps and Epps (1976) and Tauchen and Pitts (1983), have focused on this positive contemporaneous correlation, more recent analyses by Lamoureux and Lastrapes (1994) and Andersen (1996) have sought to more fully exploit the dynamic implications of the MDH by explicitly parameterizing the process for $K_{j, t}$. These parameterizations have invariably relied on fairly simple low-order autoregressive moving average type formulations. The relatively low degree of intertemporal dependence uncovered in these studies seems at odds with the aforementioned extant time series literature documenting very persistent own temporal dependencies in both volatility and trading volume. As noted by Andersen (1996), however, it is possible that different types of "news" will affect volatility and trading volume differently, thus resulting in a more complex dynamic relationship than implied by the simple MDH with a single latent information-arrival process. For instance, the regularly scheduled releases of macroeconomic announcements may induce fairly heavy trading volume accompanied by only short-lived bursts in volatility. Similarly, option expiration days might also be associated with heightened trading volume without any increase in volatility. Conversely, earnings or dividend announcements may result in dramatic price adjustments accompanied by relatively little trading activity. Motivated by these observations, Liesenfeld (1998a,b) studied these different types of "news" arrivals by using a bivariate mixture model with two information-arrival variables. It appears that, by forcing the same short-run dependence for the two series, the MDH model may un- derestimate the true long-run persistence. In the present analysis, we explicitly abstract from any short-run dynamics. The use of spectral estimation methods provides a particularly convenient approach for accomplishing this goal.

To motivate these developments, suppose that each day a particular piece of new "news" hits the market. Suppose also that the impact of a given day's "news" will last for a random number of subsequent days. It follows from Parke (1996) that, under reasonable assumptions about the corresponding survival probabilities, the resulting latent aggregate information-arrival process will be fractionally integrated. Specifically, let $d_{j}$ denote the order of fractional integration for stock $j$, so that $(1-L)^{d_{J}} K_{j, t} \equiv$ $\sum_{h=0}^{\infty} \Gamma\left(h-d_{j}\right) \cdot \Gamma(h+1) \cdot \Gamma\left(-d_{j}\right) K_{j, t-h}$ has a bounded spectrum across all frequencies. If $0<d_{j}<1 / 2$, the process is covariance stationary with autocorrelations that are eventually all positive and decay at the hyperbolic rate of $\tau^{2 \cdot d_{j}-1}$. Formal conditions for the equivalence between this definition of an $\mathrm{I}\left(d_{j}\right)$ process and the eventual hyperbolic decay rate of $\tau^{2 \cdot d_{j}-1}$ for the autocorrelation function were discussed by Beran (1994) and Robinson (1994a).

By a direct extension of the arguments of Andersen (1994, 1996) and Andersen and Bollerslev (1997a), it follows that this same long-run dependence carries over to any positive power transform of the absolute returns and volume as defined by the MDH in Equations (1) and (2). As a long-run proposition, the $\mathrm{MDH}$, therefore, implies that, for large $\tau$,

$$
\operatorname{corr}\left(\left|R_{j, t}\right|,\left|R_{j, t-\tau}\right|\right) \sim \tau^{2 \cdot d_{\jmath}-1}
$$

and

$$
\operatorname{corr}\left(V_{j, t}, V_{j, t-\tau}\right) \sim \tau^{2 \cdot d_{\jmath}-1} .
$$

If long-run dependencies are imputed into the aggregate latent information-arrival process through the gradual incorporation of daily "news" arrivals, it should manifest itself in the form of long-run hyperbolic decay rates in the autocorrelations of the observed trading volume and volatility series. Furthermore, the long-run decay rates should be the same across the two series.

Under additional distributional assumptions, it is possible to show that the cross-correlations between the absolute returns and trading volume should decay at the identical hyperbolic rate,

$$
\operatorname{corr}\left(\left|R_{j, t}\right|, V_{j, t-\tau}\right) \sim \operatorname{corr}\left(V_{j, t},\left|R_{j, t-\tau}\right|\right) \sim \tau^{2 d_{\jmath}-1} .
$$

This behavior of the cross-autocorrelations also underlies the semiparametric frequency-domain tests for fractional cointegration recently developed by Robinson and Marinucci (1998). Although the empirical investigation of such a relationship is beyond the scope of this article, it would be an interesting area to pursue in future research.

Instead, we turn to a discussion of the seminonparametric log-periodogram regression procedures that we employ in testing Equations (5) and (6). 


\section{FRACTIONAL INTEGRATION AND LOG-PERIODOGRAM REGRESSIONS}

To specify the various estimators and test statistics, let $X_{j, t} \equiv\left(\left|R_{j, t}\right|, V_{j, t}\right)^{\prime}$ denote the bivariate time series of absolute returns and trading volume for stock $j$. Assuming that the process is covariance stationary, the $2 \times 2$ spectral density matrix at frequency $\lambda$, say $f_{j}(\lambda)$, is then implicitly defined by

$$
\operatorname{cov}\left(X_{j, t}, X_{j, t-\tau}\right)=\int_{-\pi}^{\pi} \exp (i \cdot \tau \cdot \lambda) \cdot f_{j}(\lambda) \cdot d \lambda
$$

for $\tau=\ldots,-1,0,1, \ldots$ By definition, the first and second diagonal elements in this spectral density matrix correspond to the univariate spectral densities for the absolute returns and volume, respectively. If each of these series is fractionally integrated, it follows that, for frequencies $\lambda$ close to 0 ,

$$
\left\{f_{j}(\lambda)\right\}_{g g} \approx C_{j, g} \cdot \lambda^{-2 \cdot d_{j, g}},
$$

where $g=1,2 ; C_{j, 1}$ and $C_{j, 2}$ are two scaling constants; and the orders of integration, $d_{j, 1}$ and $d_{j, 2}$, refer to $\left|R_{j, t}\right|$ and $V_{j, t}$, respectively. This approximate log-linear relationship underlies the so-called GPH log-periodogram regression estimator originally proposed by Geweke and Porter-Hudak (1983), in which the logarithm of the sample periodogram ordinates is regressed on a constant and the lowest Fourier frequencies, $\lambda_{h}=2 \cdot \pi \cdot h / T$ for $h=1,2, \ldots, m$. The GPH estimator has been used fairly extensively throughout the literature, although Robinson (1995) only recently obtained formal proofs of its consistency and asymptotic normality. This semiparametric estimator necessarily results in an efficiency loss compared to a full information maximum likelihood or general method of moments (GMM)-based approach involving a complete distributional characteriza- tion of the $X_{j, t}$ process. At the same time, any misspecification of the short-run dynamics would render the maximum likelihood and GMM estimates difficult to interpret and may explain previous rejections of the MDH based on a fully specified latent information-arrival process, $K_{j, t}$. Furthermore, even for simple low-order fractionally integrated models, exact maximum likelihood estimation is extremely time consuming and would not be practical for the sample sizes and number of individual stocks analyzed here. By explicitly focusing on the long-run frequencies and the asymptotic relationship in Equation (9), the semiparametric log-periodogram regression estimators used herein avoid these difficulties and, as such, provide a particularly attractive inference strategy in the present context.

Of course, any test of the hypothesis of a common longrun hyperbolic decay rate necessarily requires a multivariate approach. To this end, let the sample periodogram for $\left\{X_{j, t}\right\}_{g}, g=1,2$, be denoted

$$
I_{j, g}(\lambda) \equiv(2 \cdot \pi \cdot T)^{-1} \cdot\left|\sum_{t=1}^{T}\left\{X_{j, t}\right\}_{g} \exp (i \cdot t \cdot \lambda)\right|^{2} .
$$

Following Robinson (1995), define the $(m-l) \times 2$ matrix, $Y_{j}$, with the $(k, g)$ th element equal to the log-periodogram at the $k$ th Fourier frequency; that is,

$$
\left\{Y_{j}\right\}_{k g} \equiv \log \left[I_{j, g}\left(\lambda_{k}\right)\right]
$$

where $k=l+1, l+2, \ldots, m$, and $g=1,2$. The asymptotic theory for the estimators to be defined later depends on the trimming and truncation parameters, $l$ and $m$, with both tending to infinity at a slower rate than the sample size, $T$, while the ratio $l / m$ tends to 0 . Practical optimality criteria for choosing both of these tuning parameters have proven elusive, but the rates of Lobato and Robinson (1996) and

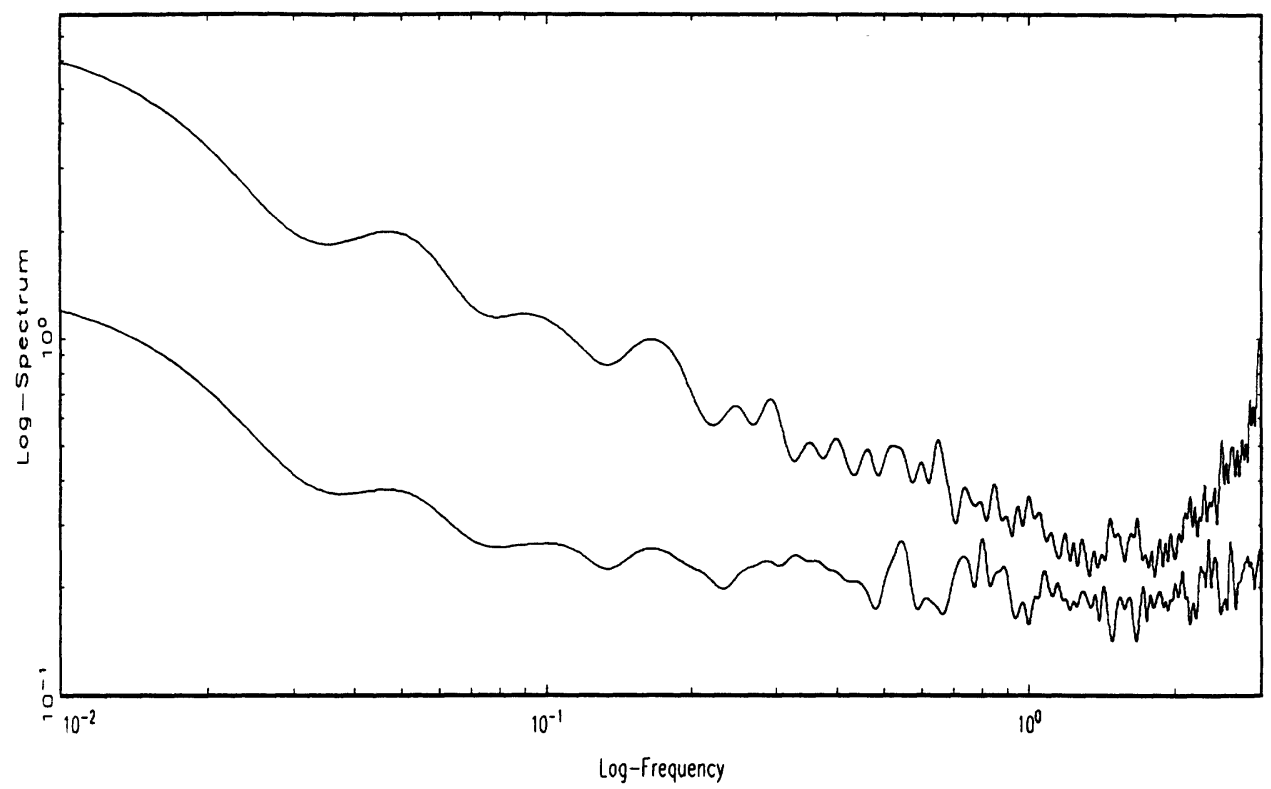

Figure 1. Log-log Spectra for the Time Series of Absolute Daily Returns and the Linearly Detrended Daily Turnover Ratios for AA. The two spectra are estimated as the smoothed-sample periodograms using a Bartlett kernel with bandwidth of $(T)^{1 / 2} \approx 91$. The sample period extends from July 2, 1962, through December 29, 1995, excluding the three-week post-October 1987 crash, for a total of 8,413 observations. The lower and upper lines correspond to the absolute returns and turnover ratios, respectively. 

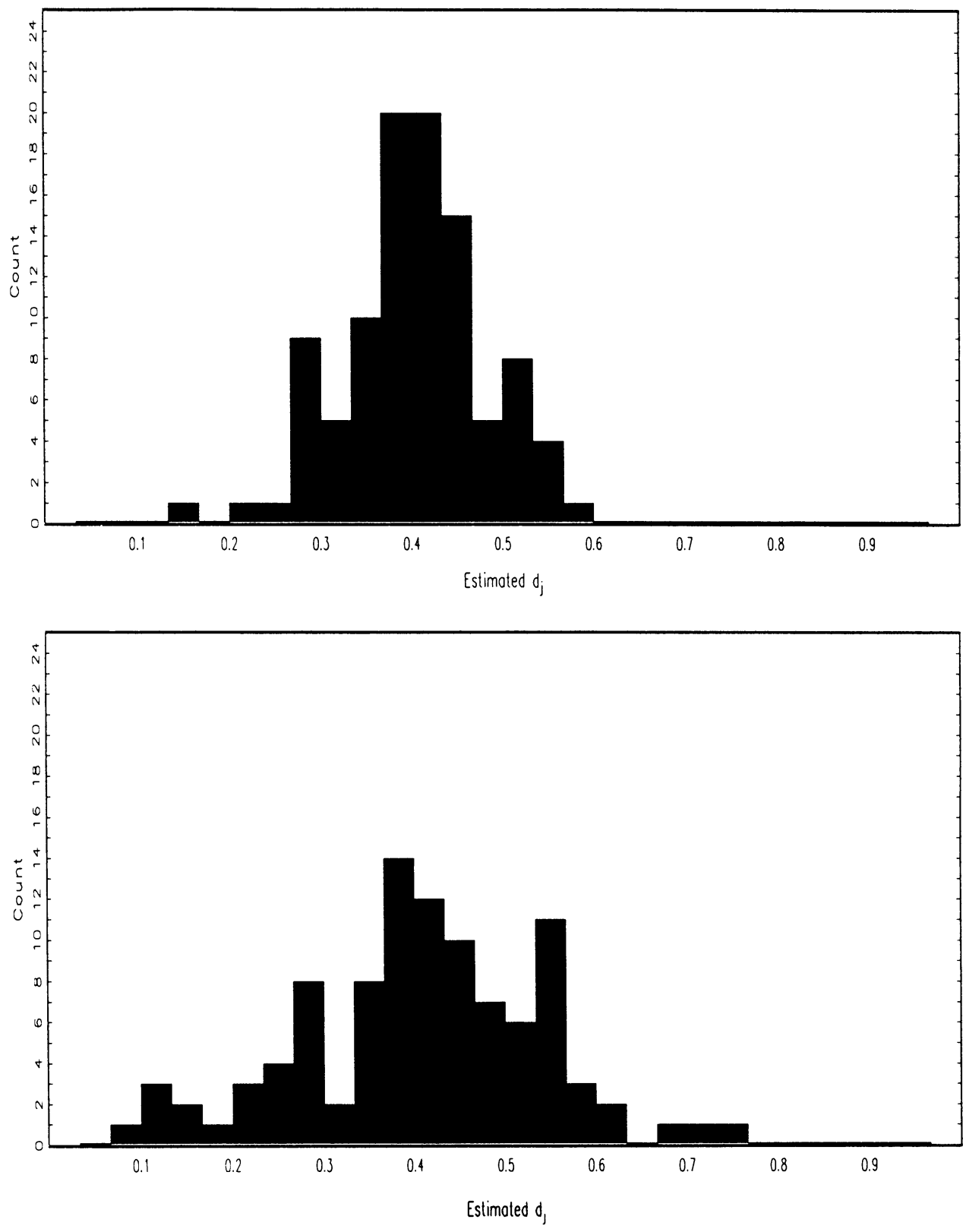

Figure 2. Estimates of Fractional Integration. Panel A gives the histogram for the 100 log-periodogram regression estimates for the degree of fractional integration for the daily absolute returns for the 100 individual common shares in the S\&P100 composite index. Panel B depicts the histogram for the 100 log-periodogram regression estimates for the degree of fractional integration for the linearly detrended daily share turnover ratios for the individual common shares in the S\&P100 composite index.

Robinson (1994b) provide useful guidance. Fortunately, the empirical results in Section 3 do not appear overly sensitive to the specific values chosen for $l$ and $m$.

Moreover, let the $2 \times 1$ vector $U_{j, k}$ be given by

$$
\left\{U_{j, k}\right\}_{g} \equiv\left\{Y_{j}\right\}_{k, g}-c_{j, g}+d_{j, g} \cdot\left[2 \cdot \log \left(\lambda_{k}\right)\right]
$$

where $c_{j, g} \equiv \log \left(C_{j, g}\right)+\psi(1)$ and $\psi(1)$, the digamma or psi function, is defined by $\left.\psi(1) \equiv(d / d z) \log \Gamma(z)\right|_{z=1} \approx$ -.577 . The $U_{j, k}$ 's are readily interpreted as the bivariate vector approximation errors associated with the relationship in Equation (11). This suggests the following least squares estimator for $d_{j} \equiv\left(d_{j, 1} ; d_{j, 2}\right)$ :

$$
\hat{d}_{j}=Y_{j}^{\prime} Z_{j}\left(Z_{j}^{\prime} Z_{j}\right)^{-1} e_{2}
$$

where $e_{2} \equiv(0,1)^{\prime}$ and the elements in the $(m-l) \times 2$ matrix of explanatory variables, $Z_{j}$, is defined by $\left\{Z_{j}\right\}_{k, 1}=1$ and $\left\{Z_{j}\right\}_{k, 2}=-2 \cdot \log \left(\lambda_{k}\right)$ for $k=l+1, l+2, \ldots, m$. For $l=0$ the two estimates for $d_{j, 1}$ and $d_{j, 2}$ correspond directly to the univariate GPH estimates. Although $\hat{d}_{j}$ may seem like a natural estimator, the corresponding $\hat{U}_{j, k}$ residual vectors do not satisfy the usual regularity conditions invoked in justifying the asymptotic properties of least squares estimates. As shown by Robinson (1995), however, under weak additional regularity conditions,

$$
\left[e_{2}^{\prime}\left(Z_{j}^{\prime} Z_{j}\right)^{-1} e_{2} \hat{\Omega}_{j}\right]^{-1 / 2}\left(\hat{d}_{j}-d_{j}\right) \rightarrow \mathrm{N}(O, I),
$$

where

$$
\hat{\Omega}_{j}=(m-l)^{-1} \cdot \sum_{k=l+1}^{m} \hat{U}_{j, k} \hat{U}_{j, k}^{\prime}
$$


This result validates conventional large-sample inference involving regression $t$ ratios and chi-squared statistics.

A consistent estimate for the standard error in the asymptotic normal distribution for $\hat{d}_{j, g}$ is given by the squareroot of the $g$ th diagonal element of the matrix in brackets in Equation (14). It is also possible to show that $\left[e_{2}^{\prime}\left(Z_{j}^{\prime} Z_{j}\right)^{-1} e_{2}\right]^{-1} \rightarrow 4 \cdot m$, and $\left.\left\{\hat{\Omega}_{j}\right\}_{g g} \rightarrow(d / d z) \psi(z)\right|_{z=1}=$ $\pi^{2} / 6 \approx 1.645$. Hence, inference regarding the individual $d_{j, g}$ 's may alternatively be based on the theoretical asymptotic standard error of $\pi \cdot(6 \cdot 4 \cdot m)^{-1 / 2} \approx .641 \cdot m^{-1 / 2}$. Similarly, a two-sided test for $d_{j, 1}=d_{j, 2}$, a common longrun hyperbolic rate of decay across the volatility and trading volume series, may be based on the asymptotic chi-squared statistic,

$$
\left(\hat{d}_{j}^{\prime} f\right)^{2} \cdot e_{2}^{\prime}\left(Z_{j}^{\prime} Z_{j}\right)^{-1} e_{2} \cdot f^{\prime} \hat{\Omega}_{j} f \rightarrow \chi_{1}^{2}
$$

where $f$ denotes the $2 \times 1$ vector $(1,-1)^{\prime}$.

With a slight abuse of notation, the corresponding restricted least squares estimator that imposes this commonality on the fractional orders of integration is most easily expressed as

$$
\hat{d}_{j}=e_{3}^{\prime}\left[Q^{\prime}\left(Z_{j}^{\prime} Z_{j} \otimes \hat{\Omega}_{j}^{-1}\right) Q\right]^{-1} Q^{\prime} \operatorname{vec}\left(\hat{\Omega}_{j}^{-1} Y_{j}^{\prime} Z_{j}\right)
$$

where $\hat{d}_{j}$ is now a scalar, $e_{3} \equiv(0,0,1)^{\prime}$, and the $4 \times 3 Q$ matrix has zeros everywhere except for ones in the $(1,1)$, $(2,2),(3,3)$, and $(4,3)$ elements. Moreover, $\otimes$ refers to the Kronecker product, and the $\operatorname{vec}(\cdot)$ operator stacks the relevant $2 \times 2$ matrix as a $4 \times 1$ vector. Like the unrestricted estimates defined in Equation (13), the $\hat{d}_{j}$ in Equation (17) is asymptotically normally distributed. The corresponding asymptotic standard error may be estimated by the square root of the last diagonal element of the matrix in brackets.

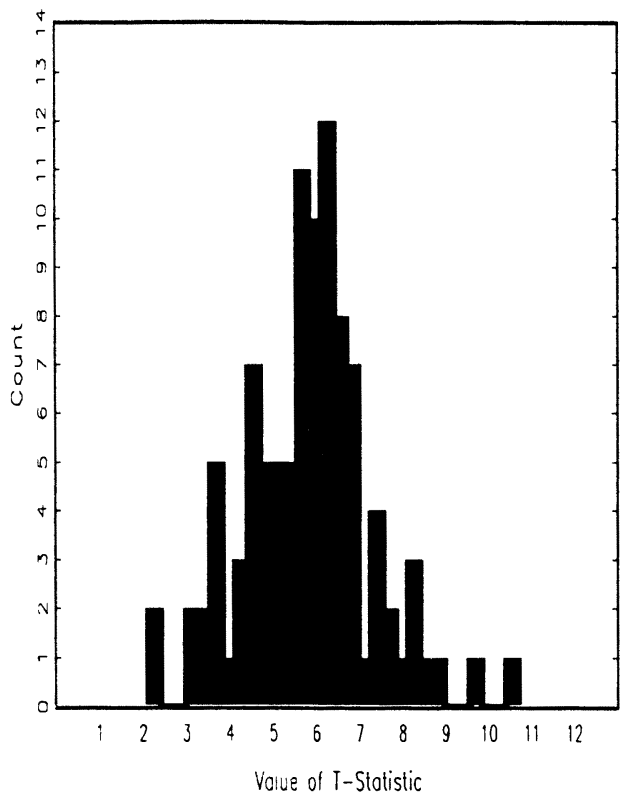

\section{ESTIMATING LONG-RUN DEPENDENCE IN EQUITY TRADING VOLUME AND VOLATILITY}

In the following section, we report the results for the estimators and test statistics defined previously when applied to the time series of returns and trading volume for the 100 individual common shares included in the S\&P100 composite index.

\subsection{Data Description}

The data consist of the bivariate absolute return and trading volume series for each of the 100 firms included in the January 10, 1997, revision of the S\&P100 broad-based composite index. Daily closing prices, trading volumes, and number of outstanding shares were obtained from the Center for Research in Security Prices database. The sample period encompasses July 2, 1962, to December 29, 1995. Following Andersen and Bollerslev (1997b) and Chan, Chan, and Karolyi (1991), we delete the three-week period immediately following the October 1987 stock-market crash. Sensitivity analysis, discussed in the Appendix, revealed the results to be materially unaffected by this omission. For the 37 companies not listed during the full-sample period, the analysis was conducted from the date of the company's first listing. The resulting minimum sample size involved 1,420 daily observations, compared to 8,440 for the full sample. However, 86 of the companies had more than 5,000 observations.

The continuously compounded returns for each of the $j=1,2, \ldots, 100$ stocks, $R_{j, t}$, were corrected for the effects of stock splits and dividends using standard procedures. Following the arguments of Lo and Wang (1996), the daily trading volume was measured by the turnover ratios $V_{j, t} \equiv S_{j, t} / N_{j, t}$, where $S_{j, t}$ denotes the share volume for stock $j$ during day $t$ and $N_{j, t}$ refers to the total number of outstanding shares at that time. Trading volume has increased dramatically over the past 30 years. To account for

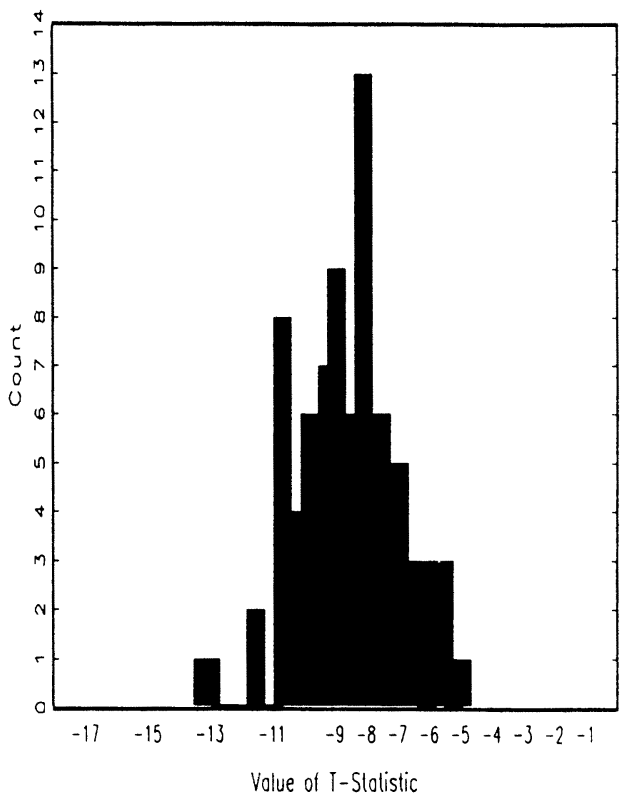

Figure 3. The Histogram for the $100 t$ Statistics for Testing the Null Hypotheses that $d_{j}=0$ and $d_{j}=1$ for the Daily Absolute Returns for the Individual Common Shares in the S\&P100 Composite Index. The $t$ tests for $d_{j}=0$ are depicted in Panel A; Panel B refers to the tests for $d_{j}=1$. 

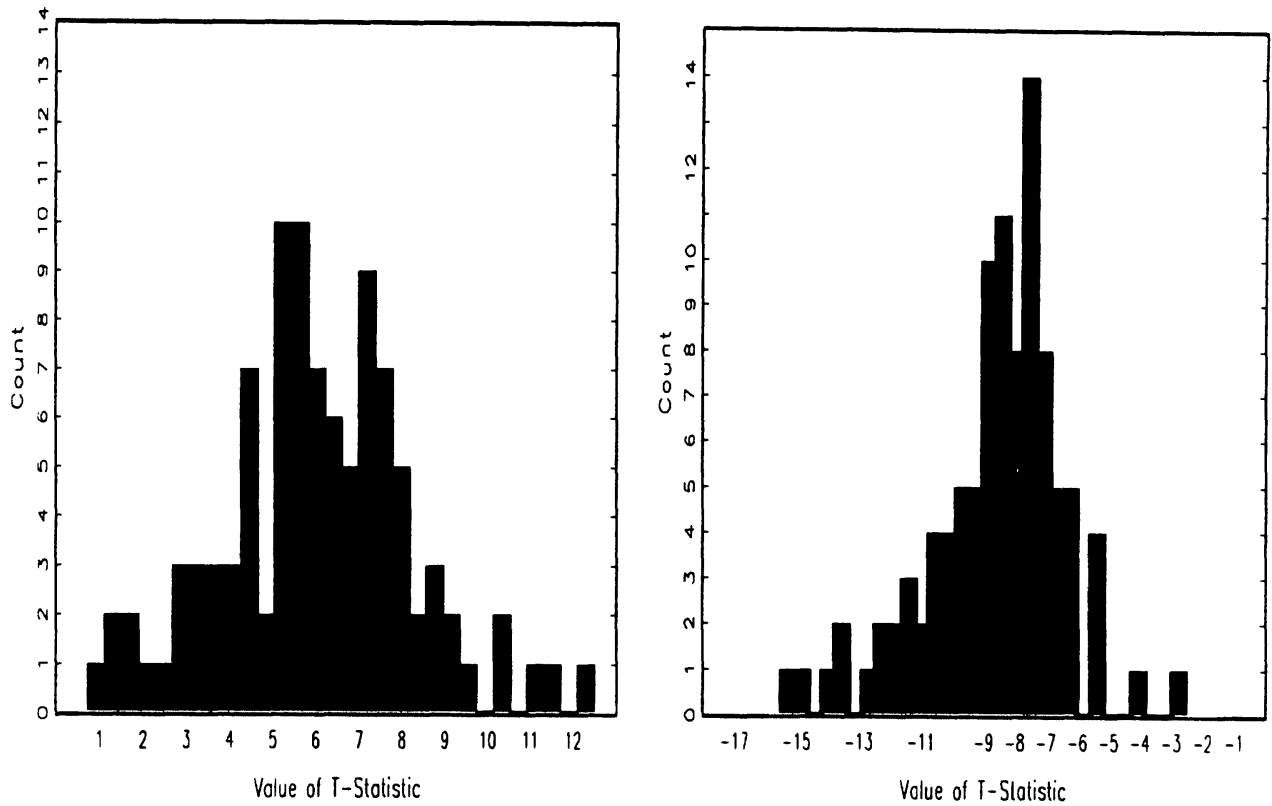

Figure 4. The Histogram for the $100 t$ Statistics for Testing the Hypotheses That $d_{j}=0$ and $d_{j}=1$ for the Linearly Detrended Daily Share Turnover Ratios for the Individual Common Shares in the S\&P100 Composite Index. The $t$ tests for $d_{j}=0$ are depicted in Panel A; Panel B refers to the tests for $d_{j}=1$.

this behavior, we extract a linear trend from all 100 volume series. More sophisticated procedures for detrending trading volume were employed by Andersen (1996) and Gallant et al. (1992), but in light of the sample sizes, the linear detrending procedure was deemed to provide a reasonable compromise between computational ease and effectiveness. In fact, neither the detrending method nor the actual pro-

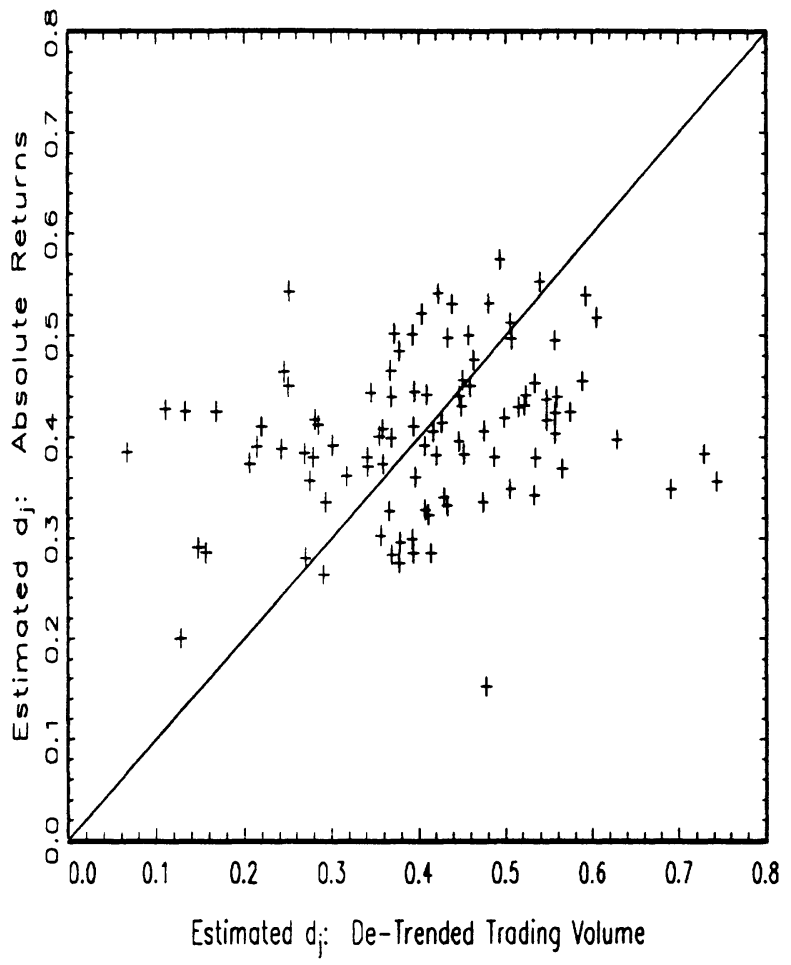

Figure 5. The Scatterplot of the 100 Bivariate Log-Periodogram Regression Estimates for the Degree of Fractional Integration for the Daily Absolute Returns and the Linearly Detrended Share Turnover Ratios for the Individual Common Shares in the S\&P100 Composite Index. cess of detrending affected any of the qualitative findings. As detailed in the Appendix, the results for the raw volume data are almost identical to those reported for the linearly detrended volume series.

\subsection{Individual Volatility and Volume Estimates}

This section summarizes the unrestricted estimates based on Equation (13). The first half of the section provides a more detailed description of the results for the Aluminum Corporation of America (AA), the alphabetically first, by ticker symbol, firm in the S\&P100 composite index. The results for the remaining stocks in the S\&P100 are discussed in the latter half of the section. All of the estimates are based on $l=1$ and $m_{j}=\left(T_{j}\right)^{1 / 2}$, where $T_{j}$ refers to the number of time series observations for firm $j$. Corroborating evidence, using $m_{j}=.5 \cdot\left(T_{j}\right)^{1 / 2}$ and $m_{j}=1.5 \cdot\left(T_{j}\right)^{1 / 2}$, for all of the firms in the S\&P100 index, is reported in the Appendix.

The data for AA covers the full July 2, 1962, to December 29,1995 , sample period for a total of 8,413 daily observations. The trading volume and absolute return series both exhibit significant excess kurtosis, with sample values of 14.44 and 15.68, respectively. The Ljung-Box portmanteau test statistics for twentieth-order own serial correlation equal 1,050 for the absolute returns and 46,320 for the trading-volume series. Clearly, both series possess a tremendous degree of own serial dependence. The corresponding unrestricted estimates for the degrees of fractional integration are $.379(.068)$ and $.534(.061)$, respectively. Based on the asymptotic standard errors reported in parentheses, both estimates are statistically greater than 0 and less than 1 at any reasonable significance level.

Note that the proof of Robinson (1995) is only valid when $-.5<d_{j}<.5$. The point estimate of $d_{j, 2}$ for AA is obviously outside of this stationary region. The stan- 
dard approach for dealing with this issue is to difference the series and then reestimate the degree of fractional integration as $\hat{d}_{j}^{*}+1$, where $\hat{d}_{j}^{*}$ refers to the estimate for the differenced series. Because the resulting numerical estimates are generally very close and relatively few of our estimates exceed .5 , we only report the estimates for the raw series.

Further justification for the notion of fractional integration is provided by Figure 1 (p. 12), which depicts the spectra of the absolute returns and the share turnover ratios for AA. The spectrum is estimated by a smoothed-sample periodogram using Bartlett weights and a bandwidth of $(T)^{1 / 2} \approx 91$. For the lowest Fourier frequencies, the spectra are both log-linear, as implied by Equation (9). The two spectra also appear quite similar over the lowest frequencies, with closely related spectral peaks and troughs. Meanwhile, the spectra look quite different at higher frequencies, indicating the existence of different short-run dynamics.

Comparable results for all of the 100 firms listed on the S\&P100 are contained in Figures 2, 3, and 4 (pp. 13, 14, and 15). Figure 2 shows the individual estimates for the degrees of fractional integration for the absolute returns and the linearly detrended trading volume. All of the estimates for the absolute returns in Panel A of the figure lie within the range of .152 to .574 , with a mean value of .404 and a median value of .407 . These estimates are extremely close to those reported by Ray and Tsay (1998), who used a canonical correlation method to estimate the degree of volatility persistence in the absolute returns of the companies composing the S\&P500 index. Their reported mean and median values

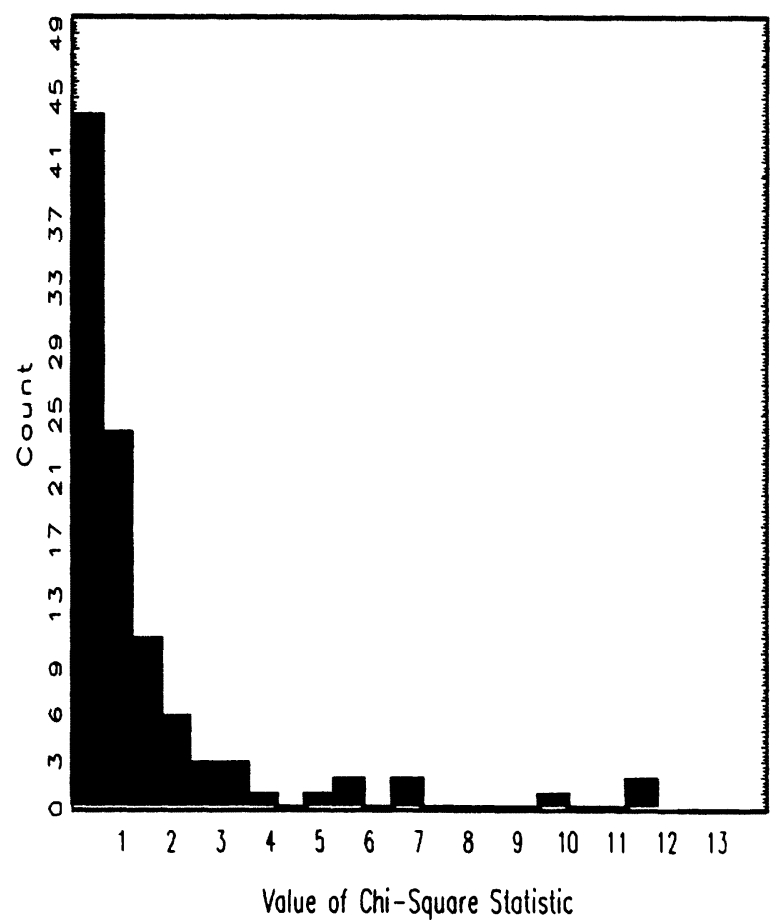

Figure 6. The Histogram for the Chi-squared Statistics for Testing Equality Between the 100 Pairwise Log-Periodogram Regression Estimates for the Degree of Fractional Integration for the Daily Absolute Returns and the Linearly Detrended Share Turnover Ratios for the Individual Common Shares in the S\&P100 Composite Index. are .404 and .395 , respectively. Figure 2, Panel B, presents the same types of histograms for the 100 individual firms' detrended trading-volume measures. These estimates range from a low of .067 to a high of .744, with mean and median values of .407 and .410 , respectively.

Figure 3, Panel A, shows the $t$ statistics for the test of the hypothesis that the individual estimates for the degree of fractional integration for the absolute returns series are greater than 0 . All of the estimated $d_{j}$ 's are, in fact, greater than 0 at the usual 5\% level, with the overwhelming majority being significant at substantially higher significance levels as well. Figure 3, Panel B, depicts the histograms for the test of the hypothesis that the individual estimates are statistically less than 1 . Again, when judged by conventional significance levels, all of the estimated $d_{j}$ 's are less than 1.

Figure 4 presents analogous $t$ tests for detrended trading volume. All but 5 of the 100 estimates are statistically greater than 0 and less than 1 at the usual $5 \%$ level. These results indicate, quite emphatically, that all the individual volatility and trading-volume series are fractionally integrated, with the vast majority of the point estimates lying between 0 and $\frac{1}{2}$.

\subsection{Common Long-Run Dependence}

The aforementioned commonality in the spectra of the volume-volatility series for AA, depicted in Figure 1, and the close correspondence between the estimates for the degree of fractional integration are highly suggestive of a common long-run hyperbolic decay rate across the two series. A formal test for this hypothesis is available in Equation (16). The value of this test statistic for AA is 2.136. Therefore, when judged by the conventional $5 \%$ chi-squared critical value of 3.841 , the test does not reject the null hypothesis that $d_{j, 1}=d_{j, 2}$. Restricting the value of $d_{j}$ to be the same across the trading-volume and volatility series, as in Equation (17), results in an estimate of $\hat{d}_{j}=$ $.465(.047)$. The much lower asymptotic standard error also indicates a nontrivial efficiency gain compared to each of the individual estimates of $d_{j}$ discussed in Section 3.2.

Results for the remainder of the firms in the S\&P100 composite index are summarized in Figures 5, 6, 7, and 8. If the long-run behavior of trading volume and volatility is truly driven by the response to a common underlying latent information-arrival process, we would expect to see a close correspondence between the estimated degrees of fractional integration for the two series. This is indeed the case. Figure 5 depicts a scatterplot of the pairwise estimates of $\left(d_{j, 1}, d_{j, 2}\right)$ for all of the 100 firms. Although there are outliers, the majority of these estimates are close to the 45-degree line.

Formal chi-squared tests for the hypothesis that the degrees of fractional integration are the same across the absolute return and trading volume series are depicted in Figure 6. Of the 100 common shares in the S\&P100 composite index, 92 produce chi-squared statistics that are smaller than the 5\% chi-squared critical value of 3.841. Although 100 test statistics are obviously not in- 


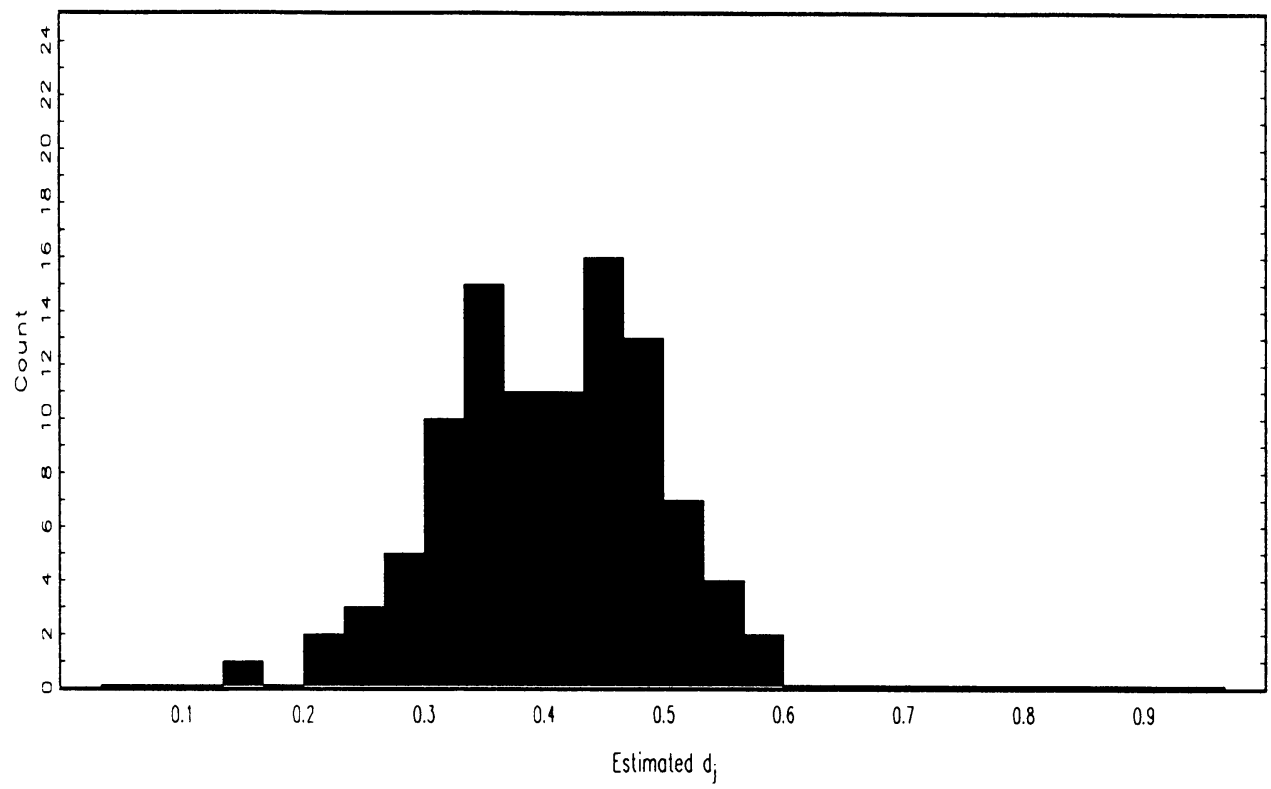

Figure 7. The Histogram for the 100 Log-Periodogram Regression Estimates Restricting the Degree of Fractional Integration for the Daily Absolute Returns and the Linearly Detrended Daily Share Turnover Ratios to Be the Same for All of the Individual Common Shares in the S\&P100 Composite Index.

dependent across the firms, the evidence of Liesenfeld (1998b) suggests that the more persistent mixture components are more closely related to firm-specific, as opposed to marketwide, phenomena. As such, each of the individual tests does provide genuine new information. Moreover, as reported in the Appendix, an examination of the eight companies for which the null hypothesis is rejected does not indicate the presence of any obvious systematic influences.

Figure 7 graphs the restricted estimates for the degree of fractional integration based on the formula in (17). Directly in line with the markedly lower standard error of $\hat{d}_{j}$ for AA noted previously, formally restrict-

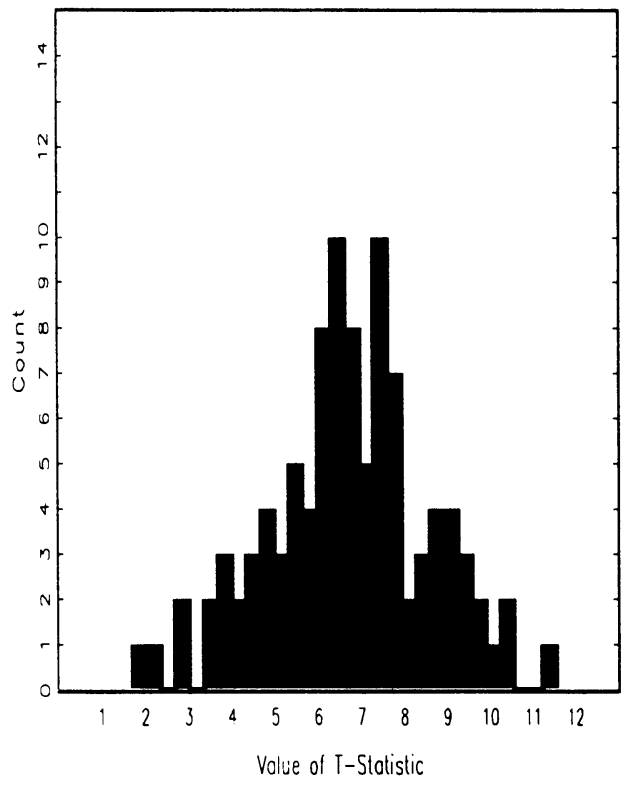

ing the $d_{j}$ 's to be the same across the two series significantly tightens the distribution of the estimates, relative to the histograms displayed in Figure 2. The values for the restricted $\hat{d}_{j}$ 's now lie within the range of .166 to .579 , with mean and median values of .406 and .407 , respectively.

Histograms of the $t$ tests for the restricted $\hat{d}_{j}$ 's, with nulls identical to those in Figures 3 and 4, are displayed in Figure 8. The individual restricted estimates strongly reject the null hypotheses of either $I(0)$ or $I(1)$. A fractionally integrated, or $\mathbf{I}(d)$, process best describes the common long-run behavior of absolute returns and trading volume.

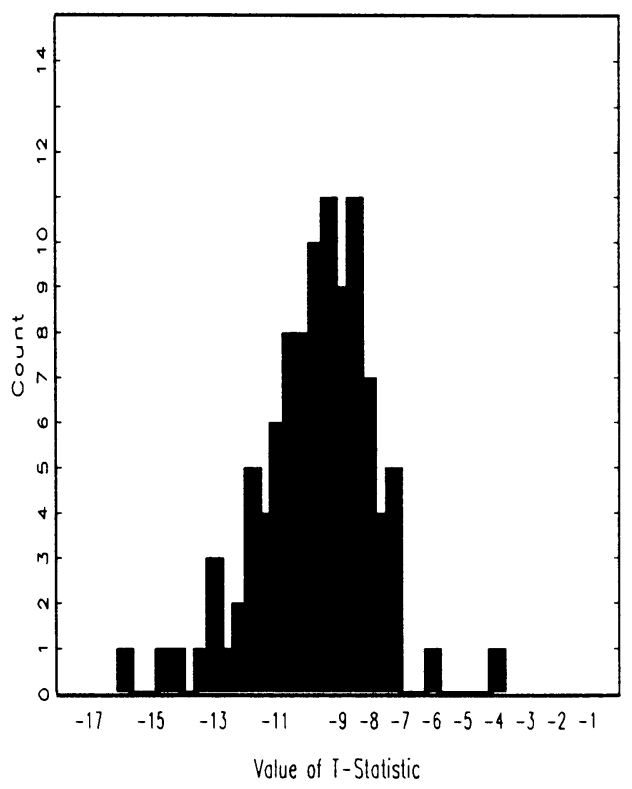

Figure 8. The Histogram for the $100 t$ Statistics for Testing the Hypotheses that $d_{j}=0$ and $d_{j}=1$ for the Restricted Estimates of the Degree of Fractional Integration for the Daily Absolute Returns and Linearly Detrended Daily Share Turnover Ratios. The $t$ tests for $d_{j}=0$ are depicted in Panel $A$; Panel $B$ refers to the tests of $d_{j}=1$. 


\section{CONCLUDING REMARKS}

This article argues that the daily returns and trading volume for the majority of the individual companies in the S\&P100 composite index are best described by meanreverting long-memory-type processes. The seminonparametric estimates and test statistics also point toward a remarkable commonality in the degree of fractional integration for each of the 100 volume-volatility pairs, with a median value of .40 . Thus, although the volume and volatility processes are both highly persistent, shocks to the two series eventually dissipate at the same slow hyperbolic rate of decay. Meanwhile, the short-run dynamics appear quite different. These empirical findings are consistent with a modified version of the $\mathrm{MDH}$, in which the volume-volatility relationship is determined by a latent information-arrival structure possessing long-memory characteristics. This may help explain the apparent rejections of the MDH reported in previous studies, which have focused on a common representation for the short-run dynamics. Instead, the results reported here suggest that allowing for differing short-lived news impacts, while imposing a common long-memory component, may provide a better characterization of the joint volume-volatility relationship in U.S. equity markets. Such a model holds the promise of improved long-run volatility forecasts and more accurate pricing of long-term financial contracts. This is also consistent with preliminary findings based on formal tests for fractional cointegration implemented using the technique of Robinson and Marinucci (1998). We leave further work along these lines for future research.

\section{ACKNOWLEDGMENTS}

Bollerslev is a Research Associate with the National Bureau of Economic Research (NBER). The research was partially supported by a grant from the National Science Foundation to the NBER and a grant from the University of Virginia. Many of the ideas in the article were stimulated by discussions with Torben G. Andersen. We are indebted to Wake Epps and Johnathan Wright for their many helpful insights. We also thank seminar participants at the University of Virginia, an associate editor, and an anonymous referee for useful comments and suggestions. Needless to say, we are solely to blame for any errors.

\section{APPENDIX: SENSITIVITY ANALYSIS}

This appendix provides sensitivity analysis of our results by implementing the estimates in Equation (13) to the raw (i.e., the trending volume) data, by including the three-week period immediately following the October 1987 crash, and by varying the truncation parameter, $m_{j}$, from our reported value of $\left(T_{j}\right)^{1 / 2}$ to $.5 \cdot\left(T_{j}\right)^{1 / 2}$ and $1.5 \cdot\left(T_{j}\right)^{1 / 2}$. Although the quantitative results vary slightly from case to case, the qualitative results do not. Absolute returns and trading volume are always fractionally integrated, and the overwhelming majority of the 100 firms share a common degree of long-run hyperbolic decay.

Figure A.1 shows scatterplots of the 100 bivariate logperiodogram regression estimates for the degree of frac- tional integration for the daily absolute returns and the linearly detrended share turnover ratios for each of the 100 firms composing the S\&P100 composite index. Panels A, C, and E are estimated without the 1987 crash, but Panels B, D, and F include the crash of October 1987. The first, second, and third rows of Figure A.1 were estimated using a bandwidth equal to $\left(T_{j}\right)^{1 / 2}, .5 \cdot\left(T_{j}\right)^{1 / 2}$, and $1.5 \cdot\left(T_{j}\right)^{1 / 2}$, respectively. Figure A.1, Panel C, is reported in the text of the article as Figure 5 but is included for comparison purposes. First, note that, although the point estimates made with the 1987 crash are slightly more dispersed, the difference across the two columns is by no means dramatic. Including the crash does not have a material effect on the estimates of the degrees of fractional integration. Increasing $m_{j}$, however, does reduce the dispersion of the point estimates. In Panel A, these estimates are in reasonable proximity to the 45-degree line, but there are a significant number of outliers. This is not unexpected because $.5 \cdot\left(T_{j}\right)^{1 / 2}$ severely restricts the number of periodogram ordinates included in the regression. As the bandwidth is increased, the number of outliers decreases and the point estimates cluster more closely around the 45-degree line. This clustering is not unduly inhibited by the inclusion of the 1987 crash.

Additional sensitivity analysis is provided in Tables A.1 and A.2. Table A.1 contains the estimates of $d_{j}$ for both trading volume and absolute returns, excluding the October 1987 crash period, and Table A.2 presents the same measures, including the October $1987 \mathrm{crash}$. The estimated values of $d_{j}$ do not significantly change between Tables A.1 and A.2. For example, the mean estimate for the degree of fractional integration for the absolute returns with $m_{j}=\left(T_{j}\right)^{1 / 2}$, excluding the $1987 \mathrm{crash}$, is .472, and including the crash produces a mean estimate of .453 . Results for the other mean, median, minimum, and maximum values are equally insensitive to the inclusion of the crash. The difference in the estimates is almost invariably in the second decimal point.

Increasing the bandwidth does have some effect on the magnitude of the point estimates. From Table A.1, the mean estimate of $d$ for the absolute returns decreases from .472 to .362 , for $.5 \cdot\left(T_{j}\right)^{1 / 2}$ and $1.5 \cdot\left(T_{j}\right)^{1 / 2}$, respectively, whereas the mean raw volume estimates decrease from .519 to .395 for these same values of $m_{j}$. Although these are substantial changes overall, they are somewhat misleading. The largest change occurs when increasing the bandwidth from $m_{j}=.5 \cdot\left(T_{j}\right)^{1 / 2}$ to $m_{j}=\left(T_{j}\right)^{1 / 2}$. The estimates remain fairly stable when the bandwidth increases from $\left(T_{j}\right)^{1 / 2}$ to $1.5\left(T_{j}\right)^{1 / 2}$.

Detrending the volume series in both Tables A.1 and A.2 reduces the magnitude of the point estimates and pulls the mean and median volume values closer to those of the absolute returns. In fact, the detrended volume series have mean and median values that are almost identical to the absolute returns, although the estimates for raw volume are more dispersed. Overall, the results appear very robust and are generally insensitive to fundamental changes in the estimation technique.

The chi-squared statistics generated from Equation (16) are equally insensitive to detrending and the inclusion or 

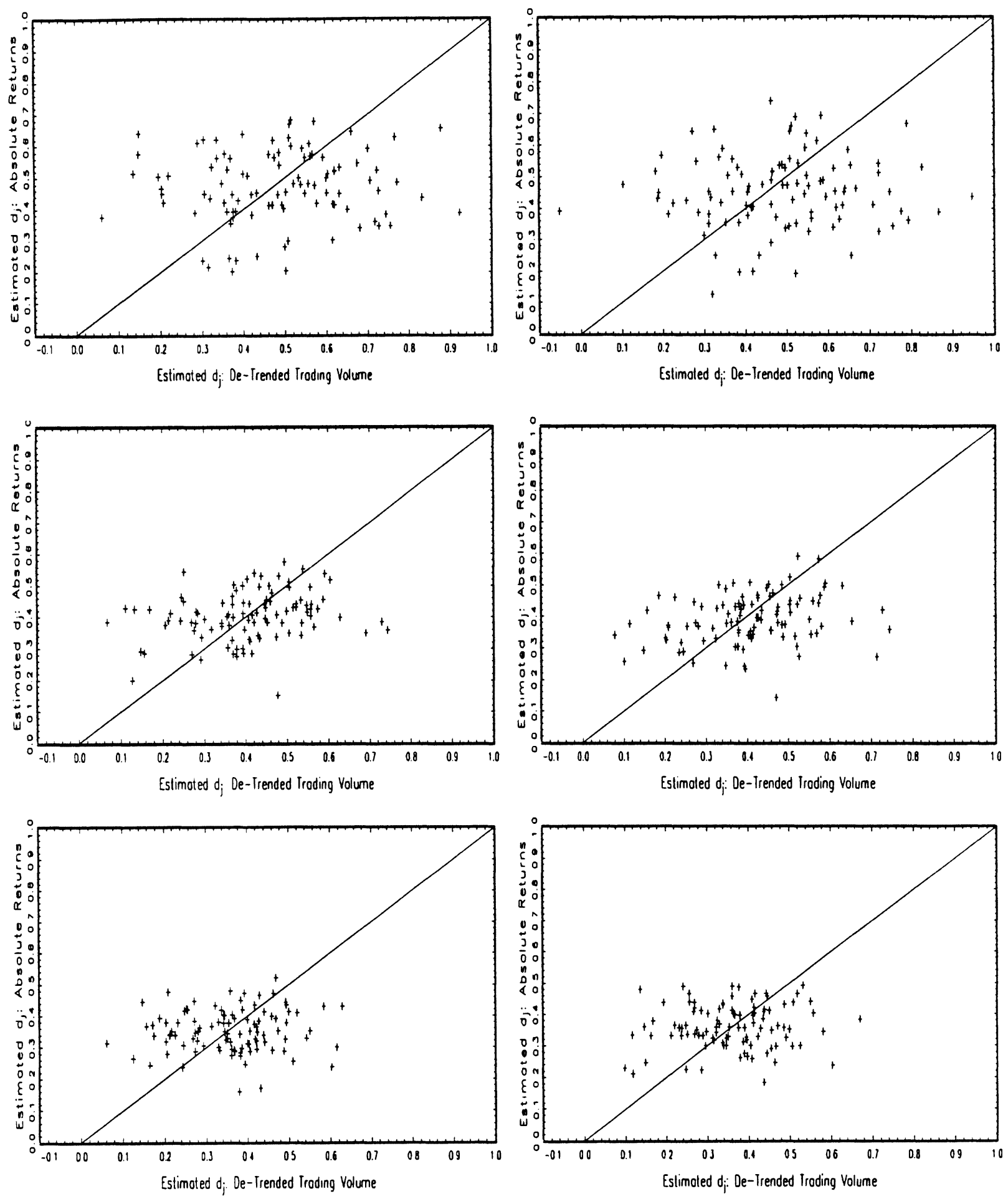

Figure A.1. catterplots of the 100 Bivariate Log-Periodogram Regression Estimates for the Degree of Fractional Integration for the Daily Absolute Returns and the Linearly Detrended Share Turnover Ratios for the Individual Common Shares in the S\&P100 Composite Index. Panels A, C, and $E$ refer to estimates omitting the three-week post-1987 crash period, whereas Panels $B, D$, and $F$ use all of the data. The estimates in Panels $A$ and $B, C$ and $D$, and $E$ and $F$ are based on bandwidth parameters of $.5 \cdot\left(T_{j}\right)^{1 / 2},\left(T_{j}\right)^{1 / 2}$, and $1.5 \cdot\left(T_{j}\right)^{1 / 2}$, respectively.

exclusion of the 1987 crash. If we allow a trend and omit the three-week post-1987 crash period, a $5 \%$ test rejects for 14 , as opposed to the reported 8 , of the 100 firms. Including the three-week period following the crash of 1987 , the number of rejections is 12 (with a trend component) and 13 (using the linear detrending method).
A detailed examination of the 8 companies that reject a common value of $d_{j}$ does not suggest the existence of any apparent systematic structure. Only 2 firms had fewer than 6,000 , or approximately 23 years worth of, observations. The 8 firms were split among retail sales (2), banking and finance (2), beverage (1), computer products (1), paper 
$\rightarrow$ Lamoureux, C. G., and Lastrapes, W. D. (1994), "Endogenous Trading Volume and Momentum in Stock Return Volatility," Journal of Business \& Economic Statistics, 12, 253-260.

$\rightarrow$ Liesenfeld, R. (1998a), "Dynamic Bivariate Mixture Models: Modeling the Behavior of Prices and Trading Volume," Journal of Business \& Economic Statistics, 16, 101-109.

(1998b), "Trading Volume and the Short and Long-Run Components of Volatility," unpublished manuscript, Eberhard-Karls University, Dept. of Economics.

Lo, A. W., and Wang, J. (1996), "Trading Volume, Part I: Definitions, Data Analysis, and Implications of Portfolio Theory," unpublished manuscript, Massachusetts Institute of Technology, Sloan School of Management.

Lobato, I. N., and Robinson, P. M. (1996), "Averaged Periodogram Estimation of Long-Memory," Journal of Econometrics, 73, 303-324.

$\rightarrow$ Lobato, I. N., and Savin, N. E. (1998), "Real and Spurious Long Memory Properties of Stock Market Data," Journal of Business \& Economic Statistics, 16, 261-268.

O'Hara, M. (1995), Market Microstructure Theory, Cambridge, MA: Basil Blackwell.

Parke, W. R. (1996), "What is a Fractional Unit Root?" unpublished manuscript, University of North Carolina, Dept. of Economics.

Ray, B. K., and Tsay, R. S. (1998), "Common Long-Range Dependence in Daily Stock Volatilities," unpublished manuscript, University of Chicago, Graduate School of Business.

$\rightarrow$ Richardson, M., and Smith, T. (1994), "A Direct Test of the Mixture of Distributions Hypothesis: Measuring the Daily Flow of Information," Journal of Financial and Quantitative Analysis, 29, 101-116.

Robinson, P. M. (1994a), "Time Series With Strong Dependence," in Advances in Econometrics: Sixth World Congress (vol. 1), ed. C. A. Sims, Cambridge, U.K.: Cambridge University Press.

(1994b), "Semiparametric Analysis of Long-Memory Time Series," The Annals of Statistics, 22, 515-539.

(1995), "Log-Periodogram Regression of Time Series With LongRange Dependence," The Annals of Statistics, 23, 1048-1072.

Robinson, P. M., and Marinucci, D. (1998), "Semiparametric Frequency Domain Analysis of Fractional Cointegration," unpublished manuscript, London School of Economics, Dept. of Econometrics and Statistics.

Robinson, P. M., and Zaffaroni, P. (1997), "Nonlinear Time Series With Long Memory: A Model for Stochastic Volatility," unpublished manuscript, London School of Economics, Dept. of Econometrics and Statistics.

$\rightarrow$ Tauchen, G. E., and Pitts, M. (1983), "The Price Variability-Volume Relationship on Speculative Markets," Econometrica, 51, 485-505.

Tauchen, G. E., Zhang, H., and Liu, M. (1996), "Volume, Volatility, and Leverage: A Dynamic Analysis," Journal of Econometrics, 74, 177-208. 